\title{
ALARGAMIENTO DE CORONA EN ZONA ESTÉTICA
}

\section{Crown lengthening in the esthetic zone}

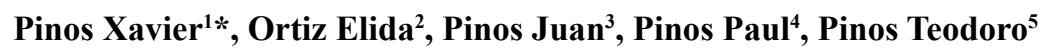 \\ ${ }^{1}$ Periodoncista - Universidad de Cuenca, Ecuador \\ ${ }^{2}$ Endodoncista - Universidad de Cuenca, Ecuador \\ ${ }^{3}$ Estudiante de Facultad de Odontología - Universidad Católica de Cuenca, Ecuador \\ ${ }^{4}$ Odontologo - Universidad Católica de Cuenca, Ecuador \\ ${ }^{5}$ Odontologo - Universidad de Cuenca, Ecuador \\ DOI: https://doi.org/10.31984/oactiva.v6i3.458
}

\begin{abstract}
Resumen
El alargamiento de corona en sector estético es un procedimiento quirúrgico periodontal que consiste en eliminar tejido gingival y óseo para crear una corona clínica más larga y desplazar el margen gingival en sentido apical obteniendo resultados estéticos a largo plazo. El objetivo de este artículo es presentar el manejo clínico del alargamiento de corona y el procedimiento restaurativo en el sector anterior para favorecer la estética en pacientes con coronas clínicas cortas y con una línea de sonrisa alta.
\end{abstract}

Palabras clave: Alargamiento de Corona, Espacio Biológico, Cirugía Plástica Periodontal

\begin{abstract}
Crown lengthening in the aesthetic sector is a periodontal surgical procedure that consists of removing gingival and bone tissue to create a longer clinical crown and displacing the gingival margin in an apical direction, obtaining long-term aesthetic results. The objective of this article is to present the clinical management of crown lengthening and the restorative procedure in the anterior sector to favor aesthetics in patients with short clinical crowns and a high smile line.
\end{abstract}

Key words: Crown lengthening, biological width, periodontal plastic surgery

\section{Introducción}

La estética es una parte esencial de práctica dental contemporánea, y una sonrisa agradable depende de la arquitectura del tejido gingival y de las características dentales. ${ }^{(1)}$ La cirugía periodontal se utiliza para crear simetría y armonía gingival dando un resultado estético su objetivo es proporcionar una adecuada longitud clínica de la corona y disminución de la visualización gingival. ${ }^{(2)}$ El alargamiento de corona es uno de los procedimientos quirúrgicos más comunes en la práctica periodontal aproximadamente el $10 \%{ }^{(3)}$

Las principales indicaciones de alargamiento de la corona incluye el tratamiento de caries subgingivales, fracturas de corona o raíz, erupción pasiva alterada, resorción radicular cervical y pilar clínico corto. La razón de ser de alargamiento de corona es para restablecer el espacio biológico, según Gargiulo et al. ${ }^{(4)}$, mide $2.04 \mathrm{~mm}$ correspondiente entre el epitelio de unión $(0,97 \mathrm{~mm})$ y tejido de inserción conectiva $(1,07 \mathrm{~mm})$. El éxito a largo plazo de un diente restaurado está relacionado con la 
preservación de un periodonto sano a través del respeto del espacio biológico, cualquier invasión en este espacio se representa clínicamente, con recesión gingival o bolsas periodontales. ${ }^{(5,6)}$

A continuación se reporta un caso clínico de alargamiento de corona en zona estética donde se detalla el trabajo multidisciplinario y su abordaje quirúrgico.

\section{Reporte de caso clínico}

Paciente masculino de 55 años de edad acude a la consulta odontología por presentar una sonrisa gingival (Figura 1), manifiesta que quiere realizarse un tratamiento estético en la zona anterior de los dientes, se planificó primero realizar una cirugía plástica periodontal con incisiones festoneadas en el área de los incisivos superiores de acuerdo a la proporción de la regla $\mathrm{T}$ de Gauge (figura 2) ${ }^{(7)}$, se levantó un colgajo mucoperíostico y se eliminó el collarín gingival. Se procedió a realizar osteotomía y osteoplastia con fresas multilaminas bajo irrigación constante de agua estéril dejando $2 \mathrm{~mm}$ desde el nuevo margen gingival a la cresta ósea (figura 3), después se suturó el colgajo con sutura simple, se le mandó medidas posoperatorias analgésicos (paracetamol 1 gr 2 veces al día durante 7 días y etoricoxib $120 \mathrm{mg}$. uno cada día por 7 días); enjuague bucal con clorhexidina $0,12 \%$ (15 $\mathrm{ml}$ por un minuto dos veces al día durante 1 semana); aplicación de crioterapia; dieta blanda durante la primera semana, evitar cualquier trauma mecánico en el sitio quirúrgico. Se retiró los puntos a los 15 días (Figura 4), a los 21 días se realizó una preparación dental de los incisivos centrales y laterales superiores y la colocación de provisionales (Figura 5), se fue realizando a la tercera, sexta y décima semana una modificación de los provisionales de $1 \mathrm{~mm}$ coronal al punto de contacto y se fue comparando el llenado de la papila en la zona entre 1.1 y 1.2 hasta los 6 meses (Figura 6) ${ }^{(8)}$, después se procedió a la preparación dental sin invadir el espacio biológico y posteriormente se realizó las impresiones definitivas para coronas de zirconio monolíticas y cambio de las prótesis removible superior, (Figura 7) se realizó un programa de mantenimiento y el paciente presenta sus márgenes gingivales estables a los 12 meses. (Figura 8)

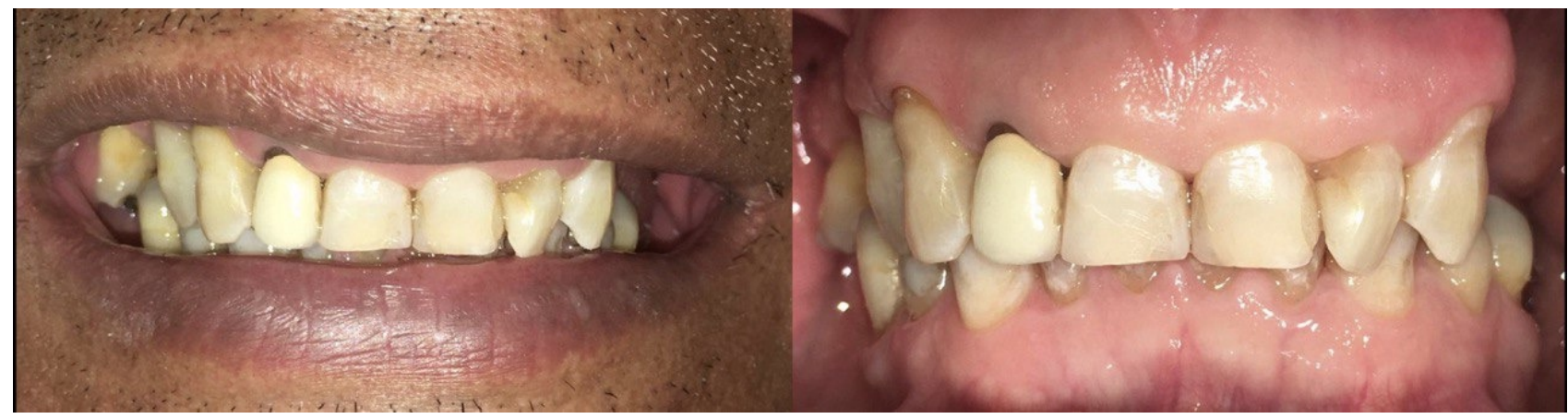

Fig. 1 Fotografía extraoral e intraoral

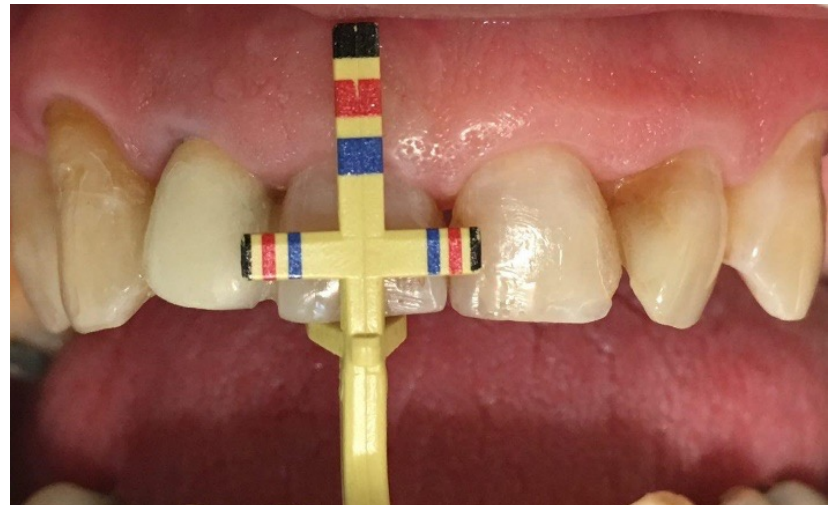

Fig. 2 T -Bar Proportion Gauge

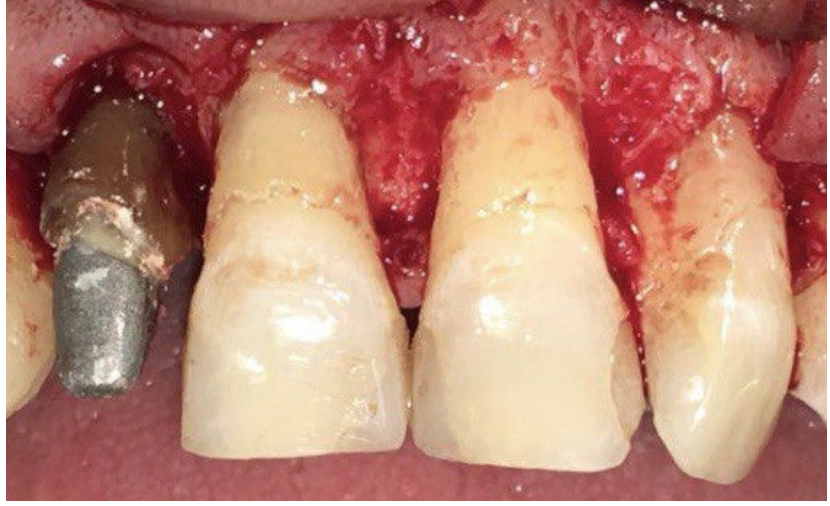

Fig. 3 Osteotomía y osteoplastia 


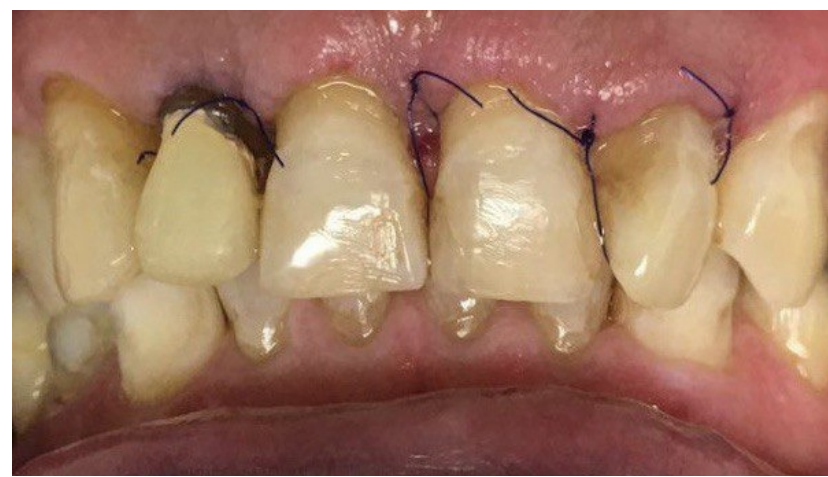

Fig. 4 retiro de puntos 15 días

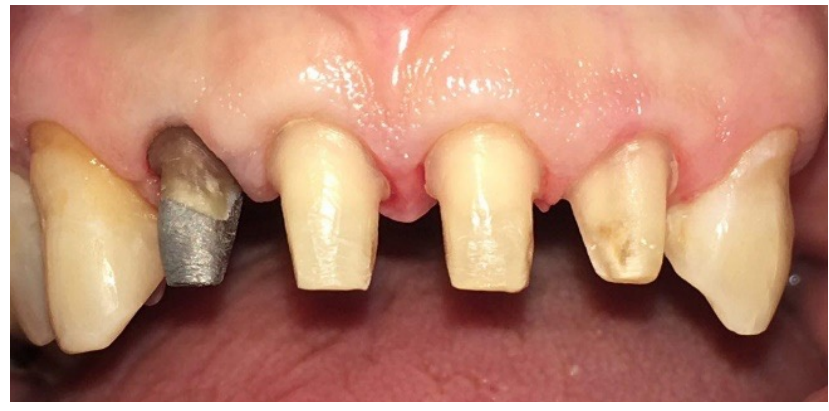

Fig. 6 Control a los 6 meses

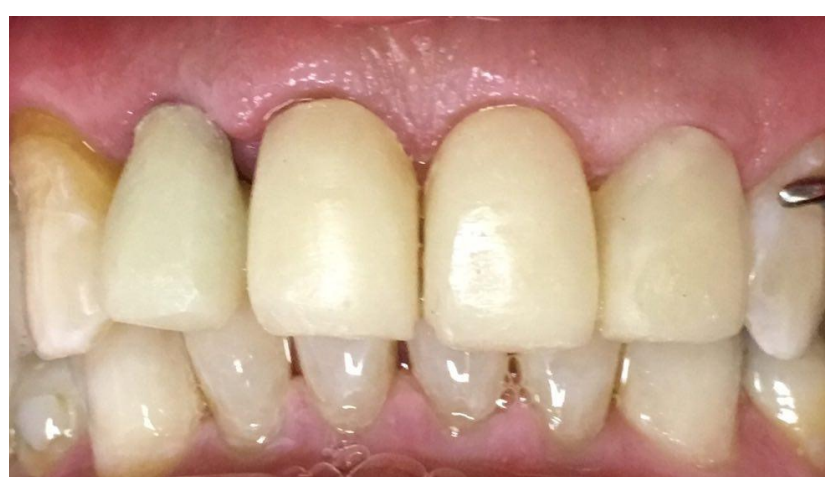

Fig. 5 Provisionales dentales

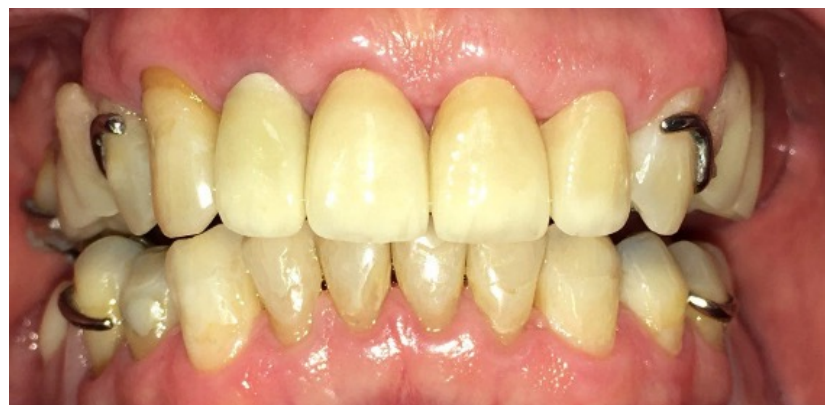

Fig. 7 Coronas de zirconio monolítico

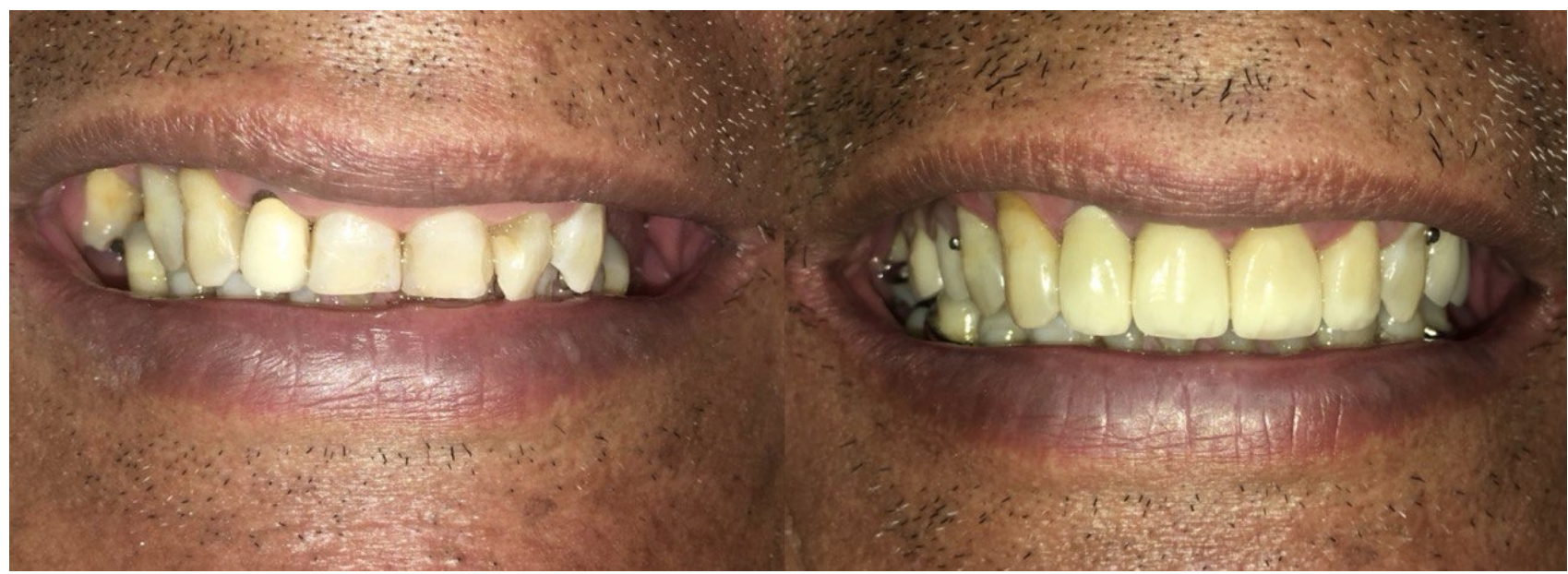

Fig. 8 Antes y después 12 meses

\section{Discusión}

La razón fundamental del alargamiento de corona clínica es restablecer el espacio biológico el cual se refiere al tejido blando que se une a la estructura dental coronal a la cresta ósea alveolar. ${ }^{(4,6)}$ Esta área compleja ha sido defendida como el único indicador de daño y descomposición del tejido cuando se invade este espacio. El valor medio del espacio biológico es de $2.04 \mathrm{~mm}$ se basó en una descripción histológica realizada por Gargiulo et al. quien describió las dimensiones basándose en 30 muestras de autopsia con 287 dientes individuales que informaron las siguientes dimensiones medias: una profundidad de surco de $0,69 \mathrm{~mm}$, epitelio de unión $0,97 \mathrm{~mm}$ y una inserción 
conectiva de $1,07 \mathrm{~mm} .^{(4)}$ En contraste al estudio anterior Vacek et al. realizaron una muestra de cadáver utilizando secciones de bloque humano no descalcificadas utilizaron 171 superficies dentales e informaron una media de 1.91 $\mathrm{mm}$ del espacio biológico (1.34 $\mathrm{mm}$ profundidad del surco, $1.14 \mathrm{~mm}$ para la epitelio de unión y $0.77 \mathrm{~mm}$ inserción conectiva). ${ }^{(9)}$ Esta es la razón por la cual en nuestro caso clínico se dejó una dimensión de $2 \mathrm{~mm}$ de espacio biológico.

Basado en observaciones histológicas en material de autopsia de perros y humanos, Wearhaug descubrió que cuando el margen restaurador de la corona se encuentra a una distancia superior a $0.4 \mathrm{~mm}$ del fondo del surco, incide en el aparato de fijación gingival e invade el espacio biológico causando la resorción ósea, que se vuelve más severa en caso de hueso cortical delgado. ${ }^{(10)}$ En cambio Nevins y Skurow recomendaron limitar la extensión del margen subgingival de 0.5 a $1 \mathrm{~mm}$ para evitar la interrupción de las uniones del tejido conectivo durante la preparación y la toma de impresión. ${ }^{(11)}$

Después de realizada una cirugía de alargamiento de corona clínica se recomienda esperar al menos 6 meses para realizar la fase definitiva del tratamiento protésico para obtener resultados restauradores estables. ${ }^{(12)}$

\section{Conclusión}

El procedimiento de alargamiento de corona clínica en sector estético tiene muy buenos resultados a largo plazo si respetamos tanto el espacio biológico, el tiempo de la fase de restauración provisional protésica después de la cirugía (3 semanas) y la fase final de la restauración protésica (6 meses).

\section{Bibliografía}

1. Liu X, Yu J, Zhou J, Tan J. A digitally guided dual technique for both gingival and bone resection during crown lengthening surgery. J Prosthet Dent. 2018;119:345-9.

2. Hempton TJ, Dominici JT. Contemporary crown-lengthening therapy: a review. J Am Dent Assoc 1939. 2010;141:647-55.

3. American Academy of Periodontology. 2003 Practice profile survey: characteristics and trends in private periodontal practice. Chicago: American Academy of Periodontology, 2004
4. Gargiulo AW, Wentz FM, Orban B. Dimensions and Relations of the Dentogingival Junction in Humans. The Journal of Periodontology. 1961

5. Hamasni FM, El Hajj F. Comparison of the Clinical Biological Width with the Published Standard Histologic Mean Values. J Int Soc Prev Community Dent. 2017;7:264-71.

6. Marzadori M, Stefanini M, Sangiorgi M, Mounssif I, Monaco C, Zucchelli G. Crown lengthening and restorative procedures in the esthetic zone. Periodontol 2000. 2018;77:84-92.

7. Fletcher P. Biologic rationale of esthetic crown lengthening using innovative proportion gauges. Int $\mathrm{J}$ Periodontics Restorative Dent. 2011;31:523-32.

8. Zucchelli G, Mazzotti C, Monaco C. A Standardized Approach for the Early Restorative Phase After Esthetic Crown-Lengthening Surgery. Int J Periodontics Restorative Dent. 2015;35:601-11.

9. Vacek JS, Gher ME, Assad DA, Richardson AC, Giambarresi LI. The dimensions of the human dentogingival junction. Int $\mathrm{J}$ Periodontics Restorative Dent. 1994;14:154-65.

10. Wearhaug J. Reacciones tisulares alrededor de coronas artificiales. J Periodontol 1953; 24: 172-85

11. Nevins M, Skurow HM. The intracrevicular restorative margin, the biologic width, and the maintenance of the gingival margin. Int J Periodontics Restorative Dent. 1984;4:30-49.

12. Pontoriero R, Carnevale G. Surgical crown lengthening: a 12-month clinical wound healing study. J Periodontol. 2001;72:841-8.

Recibido: 04 de enero de 2021

Aceptado: 08 de marzo de 2021 